\title{
New neutron lifetime measurements with the big gravitational trap and review of neutron lifetime data
}

\section{A. P. Serebrov¹, E .A. Kolomensky¹, A. K. Fomin¹, I. A. Krasnoschekova¹, \\ A. V. Vassiljev ${ }^{1}$, D. M. Prudnikov ${ }^{1}$, I. V. Shoka ${ }^{1}$, A. V. Chechkin 1 , \\ M. E. Chaikovskiyy , V. E. Varlamov ${ }^{1}$, S. N. Ivanov, A. N. Pirozhkov' ${ }^{1}$, \\ P. Geltenbort'ㄹ, 0. Zimmer ${ }^{2}$, T. Jenke², M. Van der Grinten ${ }^{3}$, and M. Tucker ${ }^{3}$}

${ }^{1}$ Petersburg Nuclear Physics Institute, NRC "Kurchatov Institute", RU-188300 Gatchina, Leningrad District, Russia

${ }^{2}$ Institut Max von Laue Paul Langevin, 71 avenue des Martyrs, F-38042 Grenoble Cedex 9, France 3 Science and Technology Facilities Council, Rutherford Appleton Laboratory, Harwell Campus, Didcot, Oxon, OX11 OQX, UK

\section{Abstract}

Neutron lifetime is one of the most important physical constants which determines

Corresponding Author:

A. P. Serebrov

serebrov_ap@pnpi.nrcki.ru

Received: 25 December 2017

Accepted: 2 February 2018

Published: 9 April 2018

Publishing services provided by Knowledge E

(c) A. P. Serebrov et al. This article is distributed under the terms of the Creative Commons

Attribution License, which

permits unrestricted use and

redistribution provided that the

original author and source are credited.

Selection and Peer-review under the responsibility of the ICPPA Conference Committee.

\section{G OPEN ACCESS} parameters of the weak interaction and predictions of primordial nucleosynthesis theory. In our experiment we measure the storage time of UCN in the material trap coated with a hydrogen-free fluorine-containing polymer (Fomblin grease UT-18). The stability of this coating to multiple thermal cycles between $80 \mathrm{~K}$ and $300 \mathrm{~K}$ was tested. The achieved storage time is only $1.5 \%$ less than free neutron lifetime. Using additional surface, which can be plunged into the trap to change the collision frequency of UCN with walls, we calculate free neutron lifetime by extrapolation to zero collision frequency. The result of the measurements with this new experimental setup is $\tau_{n}=881.5 \pm 0.7_{\text {stat }} \pm 0.6_{\text {syst }} s$ which is consistent with the conventional value of $880.2 \pm 1.0$ presented in Particle Data Group. In conclusion, we present an analysis of currently-available data on measurements of neutron.

\section{Introduction}

Free neutron lifetime is one of the most important physical constants and it's precise measurements are required to test modern physical theories. In Standard Model (SM) neutron decay probability is determined by the parameters of weak interaction and specifically by one of the parameters of Cabibbo-Kobayashi-Maskawa quark mixing matrix $V_{u d}$. Theoretical description of the beta decay of a free neutron is simpler than nuclear decay, so it is preferable to use this option to determine the element $V_{u d}$. But theoretical expression for neutron lifetime includes radiative corrections and hence, to test the predictions of $S M$, the accuracy of neutron lifetime have to be better than the 
accuracy of radiative corrections calculations. Currently, it means that accuracy have to be better than $1 \%$. $[1,2]$

Decay probability is also very important for primordial nucleosynthesis model [3]. For example, the distribution of primordial Helium directly depends on neutron lifetime, because its nuclei consist of neutrons, which survived until neutrons and protons started to form nuclei. But again, to test the cosmological models and compare the results with modern cosmological data we need to improve precision of neutron lifetime measurements.

\section{Theoretical conception of the experiment}

In our experiment the neutron lifetime is measured using stored UCN (ultra-cold neutrons) trapped by Earth's gravity in an open-topped vessel. UCN are neutrons with wavelength of about $1000 \AA$ and velocity of few meters per second. These neutrons have a unique property, they reflect from the material walls, having low loss probability, and hence can be stored in material trap for a very long time.[4]

The good approximation for UCN interaction with walls is the model of reflection at step function potential barrier with real and imaginary parts. The imaginary part describes the UCN losses in collisions. The height of the barrier and the ratio of real and imaginary parts are the properties of the material.

The open-topped vessel can be used as a gravitational spectrometer for UCN. Neutrons dynamic in the trap can be described as the movement in uniform gravitational potential with elastic reflections from the walls. If a neutron possesses enough energy to reach the top of the trap, then it can leave the open trap. Such neutrons we call above-barrier and we do not use them in our measurements. Those neutrons which do not possess enough energy can't leave the trap so they bounce from one wall to another until disappear due to loss in the collision or $\beta$-decay. The amount of neutrons in the trap exponentially decrease: $N(t, E)=N_{0} \exp \left(-t / \tau_{s t}(E)\right)$. The lifetime of neutron in a trap we call storage time. The probability for a neutron to disappear is the sum of decay and loss probability. If loss probability is much less than decay probability than storage time will be close to free neutron lifetime and hence we need to measure storage time and calculate the correction. In our experiment the correction is about $1.5 \%$ of the decay probability.

Measuring the amount of neutrons in two different moments, we can calculate the storage time. Loss probability consists on the loss function which describes the losses in one collision and collision frequency of UCNs $\tau_{\text {loss }}^{-1}=\mu(T, E) \nu(E)$. For step function model the temperature dependence parameter is factorized: $\tau_{\text {loss }}^{-1}=\mu(T, E) \nu(E)=$ 
$\eta(T) \gamma(E) . \eta(T)$ is temeprature dependent parameter determined by the material of the trap and $\gamma(E)$ is product of collision frequency and the energy dependent part of the loss function. We call $\eta(T)$ the loss coefficient and $\gamma(E)$ the effective collision frequency. Having these equations we can obtain the neutron lifetime by measuring storage times for two different $\gamma(E)$ at same temperature of the trap:

$$
\begin{gathered}
\tau_{1}^{-1}=\tau_{n}^{-1}+\eta \gamma_{1} ; \tau_{2}^{-1}=\tau_{n}^{-1}+\eta \gamma_{2} \\
\eta=\left(\tau_{2}^{-1}-\tau_{1}^{-1}\right) /\left(\gamma_{2}-\gamma_{1}\right) ; \\
\tau_{n}^{-1}=\tau_{1}^{-1}-\left(\tau_{2}^{-1}-\tau_{1}^{-1}\right) /\left[\gamma_{2}(E) / \gamma_{1}(E)-1\right]
\end{gathered}
$$

The method of obtaining neutron lifetime from storage times is linear extrapolation to zero collision frequency. There are two ways to vary effective collision frequency. One can use various energies or change geometry configuration to obtain new collision frequency. In our experiment we use both methods. The extrapolation of points differs in energies we call "energy extrapolation" and the extrapolation of points differs in collision frequency we call "geometry extrapolation".

\section{Experimental apparatus}

Our neutron trap is a copper half-cylinder with radius $0.7 \mathrm{~m}$ and length $2 \mathrm{~m}$. The large size of the trap enables the capture of numerous neutrons, greatly increasing statistical accuracy of every measurement. For example, in our previous experiment the trap had less than one fifth of this volume $[5,6]$. In order to vary neutron collision frequency, a special surface plunges into the trap. This surface (the insert) is a copper half cylinder without sidewalls with radius $0.6 \mathrm{~m}$ and length $1.8 \mathrm{~m}$. The insert has holes in the bottom to enable free exchange of neutrons between trap and insert volumes. Trap and insert turn independently using the joint "pipe-within-pipe" shaft and stepper motors.

The trap and insert covered by special polymer fomblin, which has a very small UCN capture probability especially at low temperatures. Hence, we use the cryogenic trap with cooling by liquid nitrogen to the temperature of $80 \mathrm{~K}$. In addition, this experiment requires high vacuum condition to exclude interaction with gas nuclei.

The apparatus consists of two manufactured nitrogen tanks. The outer tank contains the insulating vacuum. The inner tank contains vessels for liquid nitrogen and the primary experimental components: the neutron trap and the insert. When the vessels are filled with liquid nitrogen, the walls of the inner tank act as the thermal screen. Flexible pipelines guide vaporized gaseous nitrogen toward tubes attached to the trap 
and insert. This keeps the temperature of the trap and insert at $80 \mathrm{~K}$ with $1 \mathrm{~K}$ accuracy, monitored by 4 thermocouples - two for each surface. The inner and outer volumes are pumped out independently using turbomolecular pumps. Residual gas pressure in the trap volume does not exceed $2 \cdot 10^{-6}$ Torr. The proportional detector located outside the trap vessel so only the neutrons, which left the trap, can be counted.
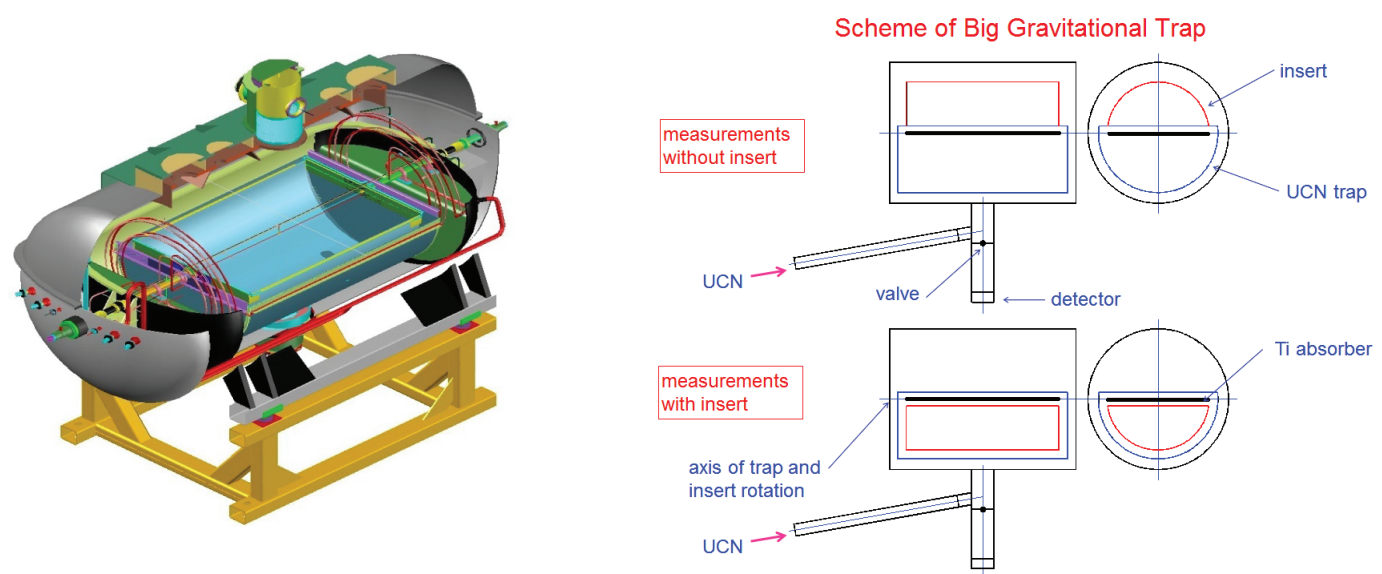

Figure 1: The scheme of the experimental apparatus.

\section{Measurement process and Monte-Carlo model}

Typical measurement cycle described below. At first, the trap turns to 90 degrees to be filled with UCN from neutron guide. In this period whole inner tank is filling with UCN. After 150 seconds, when count rate reaches plateau, neutrons are captured by turning the trap in 50 seconds to the position with 15 degrees tilt. In order to exclude above-barrier neutrons the trap holds in 15 degrees tilt position in next 500 seconds

The next step is a holding period where stored neutrons are held into the trap. Measurements with two different holding periods are required to calculate storage time and in our experiment we use 200 seconds and 1500 seconds holding periods.

After the holding period, according to theoretical concept, we need to calculate the amount of neutrons remained in the trap, hence we start the empting process. To count stored neutrons we decrease the potential barrier by turning the half cylinder trap to a specific angle. This way one can count stored neutrons with energies above new decreased barrier. Thus counted neutrons are neutrons with energies between new and initial barrier. The empting angles and the number of emptings are parameters of the experiment and we choose them using $M C$ calculations to maximize the accuracy of each measurement. The number of emptings is the number of different energy ranges for extrapolation. The last empting has 90 degrees angle which means that 
we decrease barrier to zero and count all remaining neutrons. Last 200 seconds of measurement cycle we use to measure background.

Performing measurements in various energy ranges and with two different geometry configurations, we obtain several neutron storage times, and to calculate neutron lifetime we use the extrapolation method. Corresponding effective collision frequencies have to be calculated using the $M C$ model of the experiment.

Relatively simple model of the interaction allows us to create a good MC model of the experiment. In the model, we simulate the neutron path from neutron guide to the detector.[7] The perfect agreement of experimental count rates and simulated count rates was achieved. To perform the extrapolation we have to calculate mean collision frequency in corresponding energy range. It requires energy distribution and using the MC model we reconstruct the spectrum of the UCN for every storage time and calculate mean $\gamma$.

$M C$ simulation is also useful to calculate the systematical uncertainties. We can vary parameters of the apparatus and interaction, and calculate corresponding uncertainties. During this uncertainty analysis, we discovered that geometry extrapolation is much more stable to variations of loss probability function than energy extrapolation. Therefore, we use geometry extrapolation in each measured energy region to obtain final results.

\section{Data analysis}
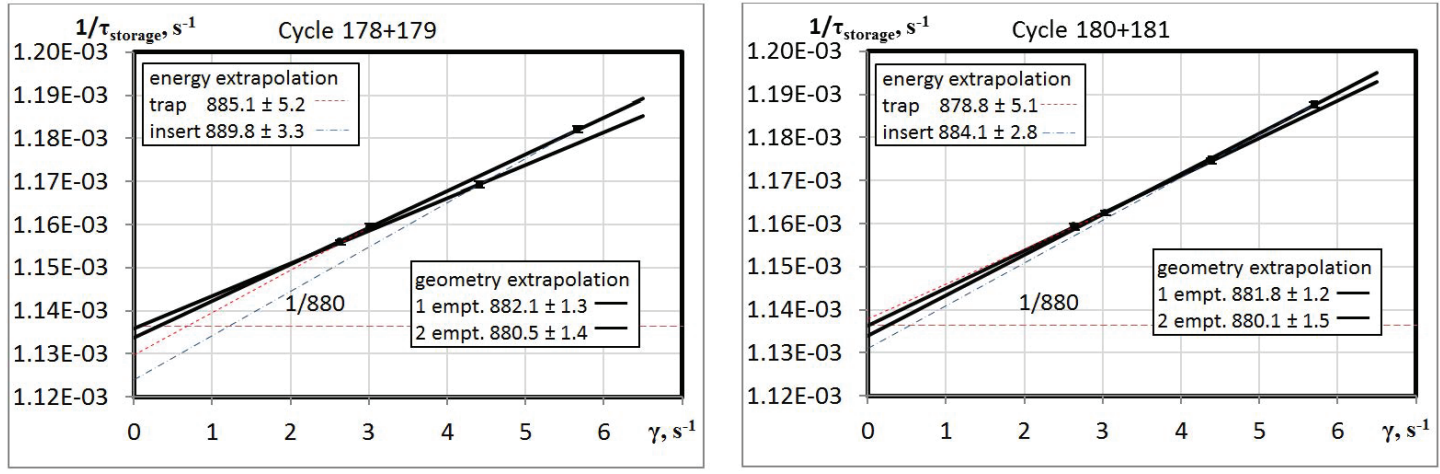

Figure 2: The results of the measurements.

The experimental apparatus is located at the Institute Laue-Langevin research reactor at the PF2 MAM UCN beam. In total, we carried out this experiment in 4 reactor cycles, which means about 200 days of data collecting. We decided to use two energy ranges 
TABLE 1: The results of the measurements.

\begin{tabular}{|c|c|c|c|}
\hline Cycle & Lower energies & Higher energies & Averaged \\
\hline $178+179$ & $880.5 \pm 1.4$ & $882.1 \pm 1.3$ & $881.4 \pm 0.9$ \\
\hline $180+181$ & $880.1 \pm 1.5$ & $881.8 \pm 1.2$ & $881.1 \pm 0.9$ \\
\hline Averaged & $880.3 \pm 1.0$ & $881.9 \pm 0.9$ & $881.3 \pm 0.7$ \\
\hline
\end{tabular}

After the second reactor cycle we improved our trap by adding titanium absorber. Using it we lowered the time required for spectrum preparation and increased the amount of useful neutrons. But we decided to separately process the data of cycles with and without absorber. All geometry extrapolation results are consistent and can be averaged to gain the free neutron lifetime. The mean value obtained after all our measurements is $\tau_{n}=881.5 \pm 0.7 \mathrm{~s}$.

To obtain final results we have to take into consideration systematical errors and corrections. To estimate the errors we use $M C$ model of the experiment and additional measurements.

\section{Conclusions}

The main advantage of this experiment is the small difference between neutron storage time in the trap and the free neutron lifetime. The large size of the UCN trap and the coating, which has small capture probability, allows us to obtain a storage time which is only 15 seconds smaller than the free neutron lifetime. The stability of the coating provides an opportunity to carry out long term experiments with long periods of gathering data. Simulation of the complete neutron paths inside the experimental apparatus allows us to increase the accuracy of the experiment by researching the systematic effects.

After analysis of all the data, we obtain value of the free neutron lifetime:

$$
\tau_{n}=881.5 \pm 0.7_{\text {stat }} \pm 0.6_{\text {syst }} s
$$

In the last 20 years, the results of several experiments aimed at measuring the neutron lifetime were published.[5,6,8-13] The technology of measuring neutron lifetime using the method of trapped UCN has progressed significantly and provided an opportunity to reach better measurement accuracy. Considering this fact, we would like to present an analysis of the results of those most recent measurements. Taking into consideration the latest results, averaged over various experiments value for the neutron lifetime is $\tau_{n}=879.5 \pm 0.8 \mathrm{~s}$. 
For better understanding, we put all these results on one diagram (Fig.3) and calculated the probability distribution for value of neutron lifetime. The well-known problem of discrepancy of beam and storage experiments remains, mostly because there is almost no new results from the beam experiments.

Analyzing the storage experiments results, although current results of storage experiments have some dispersion, one can conclude that there is no evident contradiction, and all the results are in agreement within two standard deviations accuracy, if we consider linearly summation of systematic errors. That means we need to further improve the methods of measuring neutron lifetime to reach better agreement between different methods, or find contradictions. On the other hand, better accuracy is still required to test SM and cosmological models.

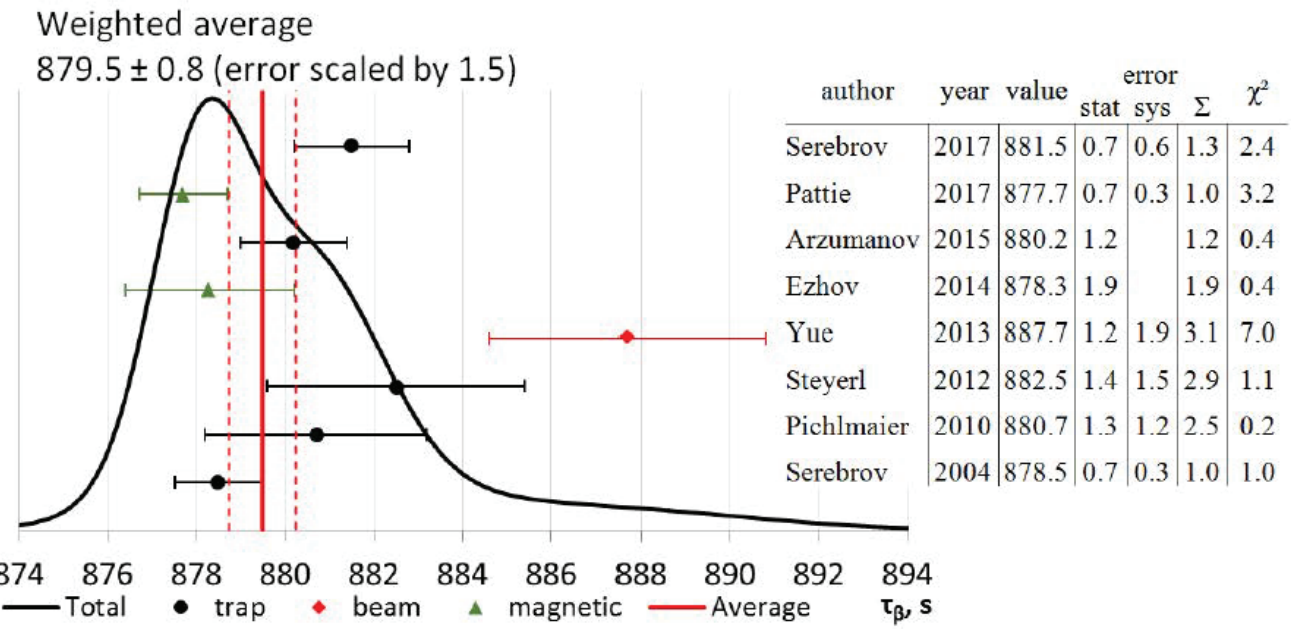

Figure 3: Distribution of results of measurements for the neutron lifetime.

\section{Acknowledgments}

The experiment was supported by Russian Science Foundation (Project № 14-22o0105)

\section{References}

[1] H. Abele et al., Eur. Phys. J. C, vol. 33, no. 1, 2004.

[2] A. Czarnecki, W. Marciano, A. Sirlin, Phys. Rev. Lett., vol. 99, p. 032003, 2007.

[3] A. V. Chechkin, A. V. Ivanchik, A. P. Serebrov, S. V. Bobashev, Tech. Phys., vol. 61, no. 7, pp. 1101-1105, 2016.

[4] V. K. Ignatovich, The Physics of Ultracold Neutrons, Oxford: Clarendon, 1990. 
[5] A. P. Serebrov, V. E. Varlamov, A. G. Kharitonov et al., Phys. Lett. B, vol. 605, pp. 72-78, 2005.

[6] A. P. Serebrov, V. E. Varlamov, A. G. Kharitonov, et al., Phys. Rev. C, vol. 78, no. 035505, pp. 1-15, 2008.

[7] A. P. Serebrov, A. K. Fomin, A. G. Kharitonov et.al., Tech. Phys., vol. 58, no. 11, pp. 1681-1687, 2013.

[8] R. W. Pattie Jr., N. B. Callahan, C. Cude-Woods et al., arXiv:1707.01817, 2017.

[9] S. Arzumanov, L. Bondarenko, S. Chernyavsky et. al, Phys. Lett. B, vol. 745, pp. 79$89,2015$.

[10] V. F. Ezhov, arxiv1412.7434, 2014.

[11] A. T. Yue, M. S. Dewey, D. M. Gilliam et al., Phys. Rev. Lett., vol. 111, no. 22, 2013.

[12] A. Steyerl, J. M. Pendlebury, C. Kaufman, et al., Phys. Rev. C, vol. 85, no. 065503, pp. 1-14, 2012.

[13] A. Pichlmaier, V. Varlamov, K. Schreckenbach, P. Geltenbort, Phys. Lett. B, vol. 693, pp. 221-226, 2010. 